\title{
Utilização da Oxigenação Extracorpórea (ECMO) na Terapia Intensiva
}

\author{
Use of Extracorporeal Oxygenation (ECMO) in Intensive Care \\ Uso de Oxigenación Extracorporeal (ECMO) en Cuidados Intensivos
}

\author{
Bianca Fonseca de Araújo \\ ORCID: https://orcid.org/0000-0003-1584-6191 \\ Centro Universitário de Patos, Brasil \\ E-mail: biancafnsca@gmail.com \\ André Mendes Figueirêdo \\ ORCID: https://orcid.org/0000-0002-0887-893X \\ Centro Universitário de Patos, Brasil \\ E-mail: andremf03@hotmail.com \\ Maria Eduarda Diógenes de Freitas Queiroz \\ ORCID: https://orcid.org/0000-0001-7062-9806 \\ Centro Universitário de Patos, Brasil \\ E-mail: mariaqueiroz@med.fiponline.edu.br \\ Saulo Landim Lucas Bezerra \\ ORCID: https://orcid.org/0000-0002-8853-954X \\ Centro Universitário de Patos, Brasil \\ E-mail: saulobezerra@med.fiponline.edu.br \\ Vitória Souza Saturnino \\ ORCID: https://orcid.org/0000-0001-6810-8657 \\ Centro Universitário de Patos, Brasil \\ E-mail: vitoriasaturnino@med.fiponline.edu.br \\ Milena Nunes Alves de Sousa \\ ORCID: https://orcid.org/0000-0001-8327-9147 \\ Centro Universitário de Patos, Brasil \\ E-mail: milenanunes@fiponline.edu.br
}

\begin{abstract}
Resumo
Objetivo: Avaliar se em pacientes em sofrimento respiratório na terapia intensiva, o uso da ECMO em comparação com os pacientes que não sofreram intervenção, melhora o prognóstico destes. Metodologia: Revisão Sistemática realizada na plataforma Medical Literature Analysis and Retrieval System Online, acessada pela Biblioteca Virtual de Saúde e no PUBMED, base de dados da U.S. National Library of Medicine. Foram utilizados os Descritores em Ciências da Saúde em inglês: Extracorporeal Membrane Oxygenation, Critical Care, Respiratory Distress Syndrome e Clinical Trial, totalizando 77 artigos. Os filtros empregados envolveram a disponibilidade do texto completo, na qual 58 artigos foram apresentados, publicação a partir do ano de 2011, atingindo 49 artigos e estudos clínicos randomizados, totalizando 10 artigos e não tendo sido encontrados documentos repetidos nas bases elegíveis. Resultados: A ECMO mostrou resultados significativos e com índices de morbimortalidade bem abaixo em comparação com os tratamentos convencionais, apesar de, em algumas situações, ela ser limitada, sendo necessário o uso de terapias adjuvantes, cuidados preventivos e intensivos que juntos resultam em uma maior chance de sobrevida e garante um aumento exponencial de melhora do prognóstico. Conclusão: Analisou-se que a terapia ECMO mostrou resultados significativos e com morbimortalidade bem abaixo de terapias convencionais. Não obstante esses resultados sejam positivos para o uso da ECMO, em algumas situações a mesma é limitada, sendo necessário o uso de terapias adjuvantes.

Palavras-chave: Oxigenação por membrana extracorpórea; Cuidados críticos; Síndrome do desconforto respiratório; Ensaio clínico.
\end{abstract}

\begin{abstract}
Objective: To evaluate whether the use of ECMO in patients with respiratory distress in intensive care, compared to patients who did not undergo intervention, improves their prognosis. Methods: Systematic review performed on the Medical Literature Analysis and Retrieval System Online platform, accessed by the Virtual Health Library and at PUBMED, database of the U.S. National Library of Medicine. Descriptors in Health Sciences in English were used: Extracorporeal Membrane Oxygenation, Critical Care, Respiratory Distress Syndrome and Clinical Trial, totaling 77 articles. The filters used involved the availability of the full text, in which 58 articles were presented, published from the year 2011, reaching 49 articles and randomized clinical studies, totaling 10 articles, and no duplicate documents were found in the eligible databases. Results: ECMO showed significant results and with morbidity and mortality rates well below compared to conventional treatments, although, in some situations, it is limited, requiring the use of adjuvant
\end{abstract}


therapies, preventive and intensive care, which together result in a greater chance of survival and guarantees an exponential increase in prognosis improvement. Conclusion: It was analyzed that ECMO therapy showed significant results and with morbidity and mortality well below conventional therapies. Although these results are positive for the use of ECMO, in some situations it is limited, requiring the use of adjuvant therapies.

Keywords: Extracorporeal membrane oxygenation; Critical care; Respiratory distress syndrome; Clinical trial.

\section{Resumen}

Objetivo: Evaluar si el uso de ECMO en pacientes con dificultad respiratoria en cuidados intensivos, en comparación con pacientes que no fueron intervenidos, mejora su pronóstico. Métodos: Revisión sistemática realizada en la plataforma Online Medical Literature Analysis and Retrieval System, a la que se accede por la Virtual Health Library y en PUBMED, base de datos de la U.S. National Library of Medicine. Se utilizaron descriptores en Ciencias de la Salud en inglés: Extracorporeal Membrane Oxygenation, Critical Care, Respiratory Distress Syndrome e Clinical Trial, totalizando 77 artículos. Los filtros utilizados involucraron la disponibilidad del texto completo, en el cual se presentaron 58 artículos, publicados desde el año 2011, llegando a 49 artículos y estudios clínicos aleatorizados, totalizando 10 artículos, y no se encontraron documentos repetidos en las bases de datos elegibles. Resultados: La ECMO mostró resultados significativos y con tasas de morbimortalidad muy por debajo de los tratamientos convencionales, aunque, en algunas situaciones, es limitada, requiriendo el uso de terapias adyuvantes, preventivas y de cuidados intensivos, que en conjunto redundan en una mayor probabilidad de supervivencia y garantías. un aumento exponencial en la mejora del pronóstico. Conclusión: Se analizó que la terapia ECMO mostró resultados significativos y con una morbimortalidad muy por debajo de las terapias convencionales. Aunque estos resultados son positivos para el uso de ECMO, en algunas situaciones es limitado, requiriendo el uso de terapias adyuvantes.

Palabras clave: Oxigenación por membrana extracorpórea; Cuidados críticos; Síndrome de dificultad respiratoria; Ensayo clínico.

\section{Introdução}

A Síndrome do Desconforto Respiratório Agudo (SARD) é um tipo de dano pulmonar inflamatório e difuso, que tem como consequência o aumento da permeabilidade vascular do tecido, bem como a diminuição da sua porção alveolar. O quadro clínico inclui achados radiológicos bilateralmente, aumento do espaço morto fisiológico, diminuição da distensão pulmonar, dispnéia, taquipnéia e hipóxia refractaria a oxigenoterapia (Cabezón et al., 2014).

Caracterizada por uma intensa deterioração na oxigenação e ventilação, a Insuficiência Respiratória Aguda Grave (SARF) é pouco frequente em adultos, em contrapartida, resulta em consequências devastadoras. Nos últimos anos, tentativas foram feitas para otimizar a ventilação protetora pulmonar. A lesão pulmonar por ventilador (VILI) é um importante fator limitante para a recuperação do parênquima, portanto, alguns pacientes com SARF refratária requerem ventilação mecânica não convencional para prevenir lesões pulmonares adicionais (Bonacchi et al., 2011).

O uso da Oxigenação Extracorpórea (ECMO) nos grupos de pacientes é avaliado a eficácia e eficiência no sistema de oxigenação. A Ventilação Mecânica Protetora com baixo volume e pressão das vias aéreas é um tratamento que salva vidas para pacientes com SARF. No entanto, em pacientes com SARF grave, o quadro de hipóxia exige alta pressão final-expiratória positiva pulmonar (PEEP), o que pode ser um desafio na oxigenação sanguínea efetiva (Mauri et al., 2016).

A Ventilação Mecânica Assistida (MV) é, de fato, reivindicada para melhorar a função dos músculos respiratórios, diminuir o risco de disfunção diafragmática, reduzir as necessidades de sedação, e favorecer o desmame das técnicas auxiliares em ventilação. Esse estudo busca, portanto, avaliar se em pacientes em sofrimento respiratório na terapia intensiva, o uso da ECMO em comparação com os pacientes que não sofreram intervenção, melhora o prognóstico destes.

\section{Metodologia}

Trata-se de um estudo de Revisão Sistemática (RS) com abordagem quantitativa. Esse tipo de abordagem, segundo Diehl (2004), objetiva a aquisição de resultados que evitem possíveis distorções de análise e interpretação e que possibilitem a maximização da margem de segurança. A RS, para Atallah e Castro (1997), consiste em um método moderno de análise 
concomitante de um conjunto de dados. Embora possa ser aplicada em várias áreas da Medicina ou Biologia, a RS é mais regularmente aproveitada na busca de evidências científicas para intervenções na saúde.

A pergunta norteadora da pesquisa, fundamentada no acrônimo PICO, foi “em pacientes em sofrimento respiratório na terapia intensiva, o uso da ECMO em comparação com os pacientes que não sofreram intervenção, traduz um melhor prognóstico?". Para a seleção e avaliação de documentos essenciais sobre a temática foi consultada a plataforma Medical Literature Analysis and Retrieval System Online (MEDLINE), acessada pela Biblioteca Virtual de Saúde (BVS) e na PUBMED, base de dados da U.S. National Library of Medicine (NLM).

Foram integrados os Descritores em Ciências da Saúde (DeCS) que consistiam em Extracorporeal Membrane Oxygenation, Critical Care, Respiratory Distress Syndrome e Clinical Trial, totalizando 77 artigos. Os filtros empregados envolviam a disponibilidade do texto completo, na qual 58 artigos foram apresentados, publicação a partir do ano de 2011, atingindo 49 artigos e estudos clínicos randomizados, totalizando 10 artigos e não tendo sido encontrados documentos repetidos nas bases elegíveis. Para a determinação e consolidação dos critérios de elegibilidade, conforme a avaliação aos pares, foi utilizado o operador booleano $A N D$, não havendo, portanto, cruzamento de operadores durante a pesquisa (Figura 1).

Figura 1: Fluxograma seletivo do material da RS, seguindo a recomendação PRISMA. Patos, PB, Brasil, 2021.

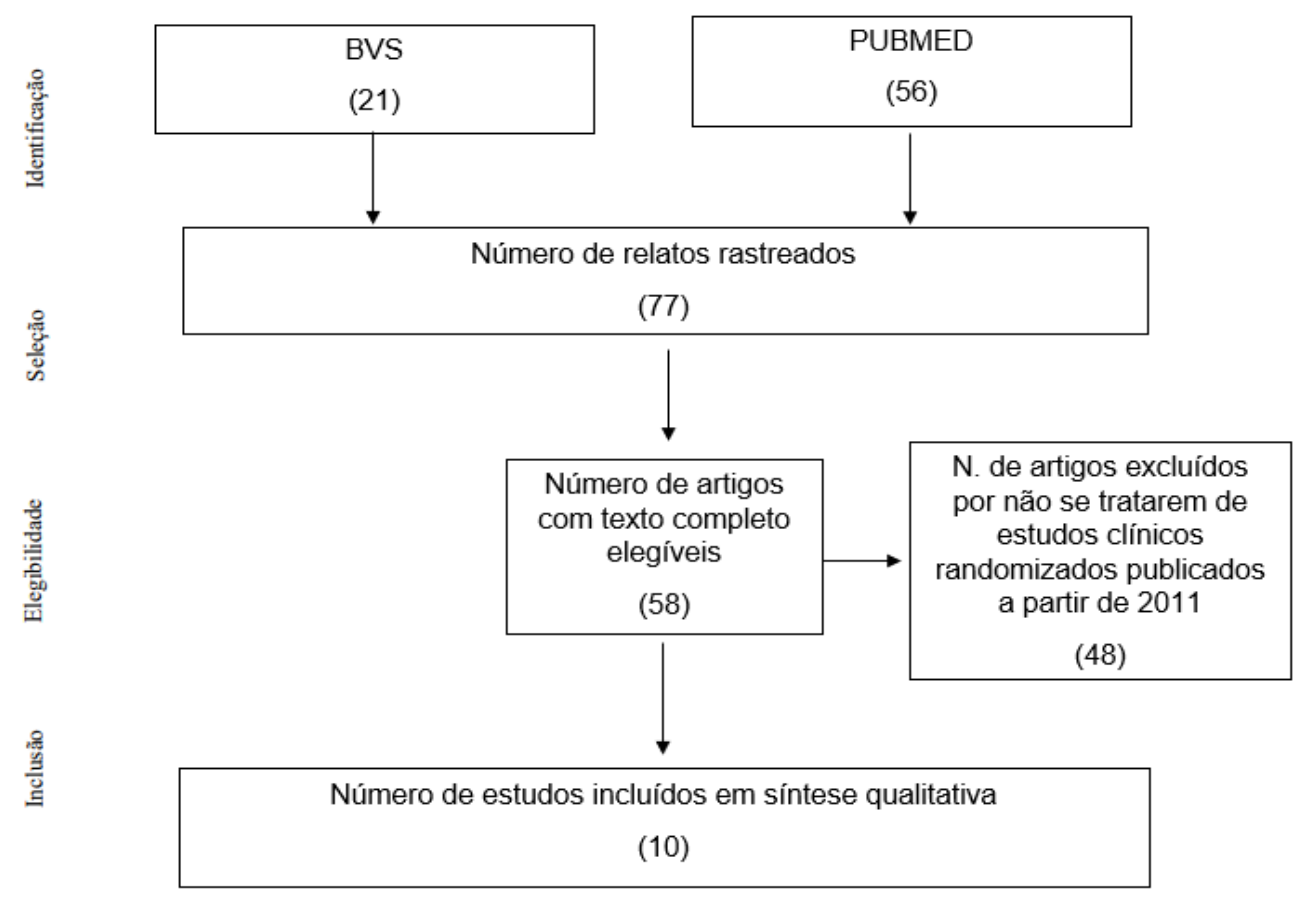

Fonte: Autoria própria (2021).

\section{Resultados}

Conforme o quadro $1,72,72 \%(\mathrm{n}=8)$ das publicações se categorizam em ensaio clínico randomizado, seguido de estudo de coorte com 18,18\% (n=2) das publicações e ensaio clínico controle 9\% (n=1). Quanto ao nível de evidência, os documentos selecionados apresentaram níveis 1,2 e 3. Quanto ao ano de publicação, houve uma distribuição igualitária em 10 anos. 
Quadro 1. Descrição dos estudos selecionados quanto aos autores/ano, tipo de estudo, nível de evidência e países. Patos, PB, Brasil, 2021.

\begin{tabular}{|l|l|l|l|}
\hline Autor/Ano & Tipo de estudo & Nível de Evidência & País \\
\hline Bonacchi et al. (2011) & Ensaio clínico randomizado & 1 & Itália \\
\hline Bronicki et al. (2015) & Estudo clínico randomizado & 1 & Estados Unidos \\
\hline Cabezón et al. $(2014)$ & Estudo de coorte & 2 & Espanha \\
\hline Combes et al. (2018) & Estudo clínico randomizado & 1 & França \\
\hline Grasselli et al. $(2019)$ & Estudo clínico randomizado & 1 & Itália \\
\hline Kim et al. (2020) & Estudo clínico randomizado & 1 & Nova Zelândia \\
\hline Mauri et al. (2016) & Ensaio clínico controlado & 3 & Itália \\
\hline Rieder et al. (2020) & Estudo clínico randomizado & 1 & Alemanha \\
\hline Schneider et al. (2017) & Ensaio clínico randomizado & 1 & Estados Unidos \\
\hline Stokes et al. (2020) & Estudo de Coorte & 2 & Estados Unidos \\
\hline
\end{tabular}

Fonte: Dados de pesquisa BVS e PUBMED (2021).

Em relação ao Quadro 2, o tratamento de paciente com a ferramenta ECMO é fundamental no avanço na medicina avançada de cuidados intensivos. No entanto, ainda chega a ser ineficaz em algumas circunstâncias, como estar atrelado ao aumento da pressão de circulação sanguínea, técnica de manuseio do paciente e cateter. Logo, apesar da eficácia, a partir da análise do quadro ficou nítida também a necessidade de aprimoramento da técnica material e do manejo dos profissionais.

Quadro 2. Autores/ano e resultados principais. Patos, PB, Brasil, 2021.

\begin{tabular}{|c|c|}
\hline Autor & Resul \\
\hline $\begin{array}{l}\text { Bonacchi et al. } \\
\text { (2011) }\end{array}$ & $\begin{array}{l}\text { Tratando-se de remoção do dióxido de carbono da circulação, os dois métodos foram semelhantes in eficácia e } \\
\text { eficiência. Sangramentos discretos foram a única complicação causada pela ECMO, a persistência desses } \\
\text { sangramentos levou } 5 \text { pacientes, } 2 \text { do grupo NS e } 3 \text { do grupo C, a receberem concentrados de hemácias. O Tempo } \\
\text { de ECMO foi mais longo nos pacientes do grupo C. Todos os pacientes do grupo NS conseguiram ECMO de } \\
\text { sucesso, contra apenas } 9 \text { do grupo C, pacientes desse mesmo grupo ainda permaneceram por mais tempo em } \\
\text { ventilação mecânica. Pacientes do grupo NS obtiveram maiores níveis de sobrevivência. }\end{array}$ \\
\hline $\begin{array}{l}\text { Bronicki et al. } \\
\text { (2015) }\end{array}$ & $\begin{array}{l}\text { Os índices médios de oxigenação (IO) basais foram } 22,0 \pm 18,4 \text { e } 25,6 \pm 14,9 \text { (grupos iNO e placebo, } \\
\text { respectivamente, } \mathrm{P}=0,27 \text { ). Houve uma tendência de melhora de OI no grupo iNO em comparação com o grupo } \\
\text { placebo em } 4 \text { horas, que se tornou significativa em } 12 \text { horas. Não houve diferença no IO entre os grupos em } 24 \\
\text { horas. Dias vivos e sem ventilação em } 28 \text { dias foi maior no grupo iNO, } 14,2 \pm 8,1 \text { e } 9,1 \pm 9,5 \text { dias (grupos iNO e } \\
\text { placebo, respectivamente, } \mathrm{P}=0,05 \text { ). Embora a sobrevida global em } 28 \text { dias não tenha alcançado significância } \\
\text { estatística, } 92 \% \text { ( } 22 \text { de } 24 \text { ) no grupo de iNO e } 72 \% \text { ( } 21 \text { de } 29 \text { ) no grupo de placebo ( } \mathrm{P}=0,07 \text { ), a taxa de membrana } \\
\text { extracorpórea livre de oxigenação a sobrevida foi significativamente maior naqueles randomizados para iNO } 92 \% \\
(22 \text { de } 24 \text { ) vs } 52 \%(15 \text { de } 29 \text { ) para aqueles que receberam placebo }(\mathrm{P}<0,01 \text { ). }\end{array}$ \\
\hline $\begin{array}{l}\text { Cabezón et al. } \\
\text { (2014) }\end{array}$ & $\begin{array}{l}\text { Métodos de menor fluxo sanguíneo, menos de } 0,51 / \text { min, que utilizam via venovenosa, permitiram diminuição de } \\
\text { aprovimadamente } 20 \% \text { dos níveis de } \mathrm{PaCO}^{2} \text { de pacientes submetidos a ventilação mecânica. O mesmo método foi } \\
\text { utilizado em pacientes com SDRA e garantiu níveis de } \mathrm{PaCO} 2 \mathrm{ypH} \text { durante a aplicação de ventilação mecânica } \\
\text { protetora }(<4 \mathrm{ml} / \mathrm{kg} \text { VT). }\end{array}$ \\
\hline
\end{tabular}




\begin{tabular}{|c|c|}
\hline $\begin{array}{l}\text { Combes et al. } \\
(2018)\end{array}$ & $\begin{array}{l}\text { Aos } 60 \text { dias, } 44 \text { dos } 124 \text { pacientes ( } 35 \% \text { ) no grupo de ECMO e } 57 \text { dos } 125 \text { (46\%) no grupo controle morreram } \\
\text { (risco relativo, } 0,76 \text {; intervalo de confiança de } 95 \% \text { [IC], } 0,55 \text { a 1,04; P = 0,09). O cruzamento para ECMO ocorreu } \\
\text { em média ( } \pm \text { DP) de } 6,5 \pm 9,7 \text { dias após a randomização em } 35 \text { pacientes ( } 28 \% \text { ) no grupo controle, com } 20 \text { desses } \\
\text { pacientes ( } 57 \% \text { ) morrendo. A frequência de complicações não diferiu significativamente entre os grupos, exceto } \\
\text { que houve mais eventos de sangramento que levaram à transfusão na ECMO grupo do que no grupo de controle } \\
\text { (em } 46 \% \text { vs. } 28 \% \text { dos pacientes; diferença de risco absoluto, } 18 \text { pontos percentuais; IC de } 95 \%, 6 \text { a } 30 \text { ), bem como } \\
\text { mais casos de trombocitopenia grave (em } 27 \% \text { vs. } 16 \% \text {; diferença de risco absoluto, } 11 \text { pontos percentuais; IC de } \\
95 \%, 0 \text { a } 21 \text { ) e menos casos de acidente vascular cerebral isquêmico (em nenhum paciente vs. } 5 \% \text {; risco absoluto } \\
\text { diferença, }-5 \text { pontos percentuais; IC de } 95 \%,-10 \text { a - } 2 \text {. }\end{array}$ \\
\hline $\begin{array}{l}\text { Grasselli et al. } \\
\text { (2019) }\end{array}$ & $\begin{array}{l}\text { O EOLIA, um ensaio clínico randomizado com pareamento da aplicação precoce de oxigenação por membrana } \\
\text { extracorpórea (ECMO) em síndrome de dificuldade respiratória aguda grave (ARDS) versus proteção } \\
\text { convencional de ventilação mecânica, não mostra estatisticamente diferença significativa ( } \mathrm{P}=0,07 \text { por log-rank } \\
\text { teste) na mortalidade em } 60 \text { dias ( } 35 \% \text { versus } 46 \% \text { em ECMO e grupo controle, respectivamente). No entanto, o } \\
\text { resultado composto, definido como passagem para ECMO ou morte em pacientes no grupo de controle e como } \\
\text { morte em pacientes no grupo ECMO, foi altamente significativa diferente (P <0,01). Sustentada também por uma } \\
\text { abordagem bayesiana para análise das tentativas do EOLIA, é possível que ECMO tem um efeito positivo no } \\
\text { resultado quando aplicado no início como um procedimento de resgate em ARDS. A ECMO provou ser } \\
\text { relativamente seguro tratamento com um perfil de segurança comparável a ventilação mecânica convencional. A } \\
\text { troca gasosa extracorpórea pode desempenhar um papel fundamental na minimização de lesão pulmonar induzida } \\
\text { pela ventilação se aplicada anteriormente num estágio menos grave da ARDS. }\end{array}$ \\
\hline Kim & $\begin{array}{l}\text { onfigurar um ventilador mecânico ainda é um grande desafio devido a variedade de pacientes, doenças e prática } \\
\text { ínica. Esse estudo busca fornecer um método para tratamento específico com a PEEP adequada para cada } \\
\text { aciente. }\end{array}$ \\
\hline al. (2016) & $\begin{array}{l}\text { Em pacientes em recuperação de SDRA submetidos à ECMO, a quantidade de dióxido de carbono removida pelo } \\
\text { pulmão artificial pode influenciar a respiração espontânea. Os efeitos da remoção do dióxido de carbono na } \\
\text { respiração espontânea durante as fases agudas iniciais da SDRA ainda precisam ser elucidados. }\end{array}$ \\
\hline $\begin{array}{l}\text { Rieder et } \\
(2020)\end{array}$ & $\begin{array}{l}\text { Em COVID-19 grave suportado com ECMO venovenosa, a adsorção de citocinas com um adsorvedor CytoSorb } \\
\text { durante as primeiras } 72 \text { horas de suporte com ECMO não resultou em concentrações reduzidas de IL-6 após } 72 \\
\text { horas. A adsorção de citocinas foi associada a um risco aumentado de mortalidade em } 30 \text { dias após o início da } \\
\text { ECMO. Portanto, a adsorção precoce de citocinas deve ser evitada em pacientes com COVID-19 que requerem } \\
\text { suporte de ECMO venovenoso. }\end{array}$ \\
\hline $\begin{array}{l}\text { Schneider et al. } \\
\text { (2017) }\end{array}$ & $\begin{array}{l}\text { Em crianças, o início do suporte de oxigenação por membrana extracorpórea está associado a sedação profunda, } \\
\text { exposição a sedativos substanciais e aumento da frequência de síndrome de abstinência iatrogênica. Um protocolo } \\
\text { de sedação padronizado, direcionado a objetivos e conduzido por enfermeiras pode ajudar a mitigar esses efeitos. }\end{array}$ \\
\hline $\begin{array}{l}\text { Stokes } \\
(2020)\end{array}$ & $\begin{array}{l}\text { Os resultados incluíram sangramento e eventos tromboembólicos, duração do suporte de oxigenação por } \\
\text { membrana extracorpórea, tempo de internação hospitalar e sobrevida hospitalar entre } 55 \text { pacientes que receberam } \\
\text { oxigenação por membrana extracorpórea venovenosa. Eventos hemorrágicos ocorreram em } 25 \text { pacientes }(45,5 \%) \text {, } \\
\text { e o tromboembolismo ocorreu em oito pacientes }(14,5 \%) \text {. Eventos hemorrágicos foram associados a maior } \\
\text { duração do suporte de oxigenação por membrana extracorpórea }(p=0,007) \text { e pior sobrevida hospitalar }(p= \\
0,02) \text {. Os eventos tromboembólicos não parecem estar associados aos desfechos clínicos. }\end{array}$ \\
\hline
\end{tabular}




\section{Discussão}

O presente estudo teve como objetivo avaliar a eficiência do uso da ECMO para o tratamento da Síndrome da Angústia Respiratória do Adulto em comparação a não intervenção.

Nesse sentido, verificou-se que o tratamento de pacientes em síndrome da angústia respiratória com ECMO tem se tornado uma ferramenta importante na medicina avançada de cuidados intensivos, no entanto, por vezes chega ser ineficaz quando não associado a técnicas adjuvantes. Os presentes estudos avaliados nesta revisão propuseram, portanto, a avaliação da eficácia da oxigenoterapia por membrana extracorpórea, que se mostraram eficazes quando associados a terapias e cuidados adjuvantes para melhora do prognóstico e diminuição da taxa de mobimortalidade dos pacientes.

Constatou-se que passados 60 dias do início da intervenção houve uma diferença de mortalidade significativa entre o grupo que recebeu o tratamento com ECMO e o que recebeu o tratamento convencional, neste último a taxa de mortalidade foi $11 \%$ maior. Também foi possível avaliar que, ao longo desses 60 dias, os pacientes em uso de ECMO foram menos submetidos à posição prona, uma diferença média de 13 dias entre os grupos. O mesmo se deu para a necessidade de hemodiálise, nesse contexto houve uma diferença média de 18 dias. Mesmo quando houve falência renal e cardíaca, esses pacientes levaram mais tempo para desenvolvê-la, com diferenças de 25 e 7 dias respectivamente (Combes et al., 2018).

No entanto, os pesquisadores constataram que falência múltipla de órgãos, parada respiratória e choque séptico foram as principais causas de morte em ambos os grupos. Os pesquisadores destacaram ainda que, mesmo com diversos benefícios, os pacientes em uso da ECMO são submetidos a riscos, neste estudo dois pacientes morreram por complicações decorrentes da instalação da terapia. Esse grupo registrou ainda maiores níveis de trombocitopenia severa que o grupo que recebeu a terapia convencional, uma diferença de 11 pontos percentuais, precisou mais de concentrado de hemácias, diferença de 18 pontos percentuais. Igualmente, os índices de choque isquêmico foram menores no grupo que recebeu ECMO, diferença de 5 pontos percentuais, contudo, os índices de choque hemorrágico foram semelhantes, bem como os de pneumotórax, pneumonia associada ao uso do ventilador, e sangramento massivo (Combes et al., 2018).

A lesão do parênquima é o principal meio de falha nesses pacientes. Os pacientes em SARF podem se beneficiar de suporte VV-ECMO e suporte para "repouso pulmonar" por redução da pressão ventilatória, concentração de oxigênio, e, como consequência, redução significativa de VILI e melhoria da recuperação pulmonar. Uma seleção cuidadosa de pacientes que tiveram a terapia com ventilador convencional sem sucesso e a aplicação de ECMO no início da doença provavelmente afetou o processo e o resultado: é importante limitar a lesão pulmonar antes e durante o suporte de ECMO para maximizar a chance de recuperação (Bonacchi et al., 2010).

Outro estudo italiano debateu pontos levantados, foi relatado que não houve diferença significativa entre a ECMO precoce e a ventilação mecânica protetiva convencional em casos de síndrome da angústia respiratória do adulto. Dentro de 60 dias a mortalidade foi semelhante, no entanto ao fim do estudo os pacientes em uso de ECMO morreram menos. Os pesquisadores relatam que é possível que a ECMO tenha um efeito positivo no desfecho, quando aplicada de forma precoce em "resgate" da SARD. Contrariando expectativas, a ECMO provou ser uma terapêutica segura mesmo quando comparada com a ventilação mecânica tradicional, e somando a isso, a troca gasosa extracorpórea minimiza lesões pulmonares induzidas por ventilador se aplicado precocemente em estágios menos severos de SARD (Grasseli et al., 2019).

Em contrapartida, os riscos da terapia com ECMO repercutem também em nível dos quadros hemorrágicos e tromboembólicos. Pacientes que tiveram um evento hemorrágico experimentaram sobrevida pior do que pacientes que não apresentaram evento de sangramento. Em contraste, os eventos tromboembólicos foram menos frequentes e não pareceram afetar a sobrevivência (Stokes et al., 2020). 
Vários fatores podem contribuir para o sangramento e tromboembolismo em pacientes recebendo ECMO. A interface do sangue e componentes não biológicos do circuito causam ativação do sistema de compressão e o consumo e degradação de fatores hemostáticos. A doença crítica subjacente aumenta os riscos de sangramento e tromboembolismo, viu-se também que a anticoagulação contínua durante a ECMO pode aumentar o risco de sangramento (Stokes et al., 2020).

Ademais, alternativas como o uso da terapia com Óxido Nítrico Inalado, foram levantados por estudo avaliado na presente revisão. Um ensaio clínico randomizado, realizado com pacientes de nove centros hospitalares de vários estados dos EUA, levou em consideração 55 crianças com síndrome da angústia respiratória aguda. O intuito da pesquisa foi testar a hipótese de que o óxido nítrico inalado (NOi) levaria à melhora da oxigenação e à diminuição da duração da ventilação mecânica em pacientes pediátricos com síndrome do desconforto respiratório agudo (Bronicki et al., 2015).

Observou-se que houve uma tendência de melhora de oxigenação no grupo iNO em comparação com o grupo placebo em 4 horas, que se tornou significativa em 12 horas. Não houve diferença no IO entre os grupos em 24 horas. Os dias de vida e sem ventilação em 28 dias foi maior no grupo iNO, 14,2 \pm 8,1 e 9,1 \pm 9,5 dias (grupos iNO e placebo, respectivamente, P = 0,05). Nesse caso, embora a sobrevida global em 28 dias não tenha alcançado significância estatística, 92\% (22 de 24) no grupo de iNO e 72\% (21 de 29) no grupo de placebo $(\mathrm{P}=0,07)$, a taxa de livre de oxigenação por membrana extracorpórea a sobrevivência foi significativamente maior naqueles randomizados para para aqueles que receberam placebo $(\mathrm{P}<0,01)$. Logo, o uso de NOi associada a técnica de ventilação mecânica apresentou resultado significativo a ser considerado em futuras práticas (Bronicki et al.,2015).

Ainda nos EUA, com mil duzentos e cinquenta e cinco pacientes, sendo essas crianças de 2 semanas a 17 anos de idade, com síndrome da angústia respiratória aguda pediátrica moderada / grave de hospitais referenciados nos estados de Massachutsetts, Oregon e Pensilvânia, foi realizado um estudo que objetivou descrever o manejo da sedação em crianças com suporte de oxigenação por membrana extracorpórea para insuficiência respiratória aguda. Na análise, sessenta e uma avaliação randomizada de titulação de sedação para insuficiência respiratória (5\%) pacientes com síndrome de angústia respiratória aguda pediátrica moderada/grave receberam suporte de oxigenação por membrana extracorpórea, incluindo 29 administrados por protocolo de avaliação aleatória de titulação de sedação para insuficiência respiratória (Schneider et al.,2017).

Assim, pacientes com oxigenação por membrana extracorpórea experimentaram mais abstinência iatrogênica clinicamente significativa do que pacientes com síndrome da angústia respiratória aguda pediátrica moderada/grave tratados sem suporte de oxigenação por membrana extracorpórea (p<0,001) (Schneider et al., 2017). Para tais autores, em crianças, o início do suporte de oxigenação por membrana extracorpórea está associado à sedação profunda, exposição a sedativos substanciais e aumento da frequência de síndrome de abstinência iatrogênica. Logo, associado à prática do ECMO, faz-se necessário um protocolo de sedação padronizado, direcionado a objetivos para garantir a eficácia do tratamento sem maiores prejuízos de abstinência para os pacientes depois.

Por fim, constatou-se ainda que ao analisar a eficiência entre a ECMO venoarterial (VA-ECMO) e a ECMO venovenosa (VV-ECMO), Bonacchi et al. (2010), recordam que embora VA-ECMO percutânea permite uma maior e mais consistente entrega de oxigênio, VV-ECMO reduz a invasividade e principais complicações relacionadas à canulação arterial, comprometimento do desempenho cardíaco, diminuição coronariana e transporte cerebral de oxigênio e autorregulação, alteração regional fluxo sanguíneo para órgão vital, aumento da resistência pulmonar, e mais.

De acordo com a avaliação das evidências feita pelo sistema Oxford Centre for Evidence-based medicine (Galvão \& Pereira, 2014), pode-se concluir que há forte recomendação em relação aos dados que foram analisados. Entretanto, nesta investigação, houve limitações relacionadas à quantidade de estudos clínicos randomizados disponíveis, embora haja grande quantidade de diversos estudos relacionados ao tema. Contudo, pode ter associação com as bases de dados pesquisadas. 


\section{Conclusão}

Constatou-se que a terapia ECMO mostrou resultados significativos e com morbimortalidade bem abaixo de terapias convencionais, além de menor necessidade de pronação e de hemodiálise em pacientes com ECMO.

Verificou-se, também, que as principais causas de mortes em quaisquer dessas terapias mantiveram-se inalteradas, sendo a falência múltipla de órgãos, parada respiratória e choque séptico as principais. Embora esses resultados sejam positivos para o uso da ECMO, em algumas situações a mesma é limitada, sendo necessário o uso de terapias adjuvantes. Levando em conta os estudos aprofundados de caso, terapias adicionais, cuidados preventivos e intensivos que juntos resultam em uma maior chance de sobrevida e garante um aumento exponencial de melhora do prognóstico.

Portanto, espera-se que novos estudos com delineamentos metodológicos mais robustos e em amostras maiores sejam desenvolvidos, buscando promover melhorias no tratamento para pacientes em sofrimento respiratório na terapia intensiva, associado ao uso da ferramenta ECMO de forma a comprovar cientificamente a melhoria da técnica associada a terapias adjuvantes.

\section{Referências}

Atallah et al. (1997). Revisão Sistemática da Literatura e Metanálise: melhor forma de evidência para tomada de decisão em saúde e a maneira mais rápida de atualização terapêutica. Diagnóstico e Tratamento, 2(2), 20-28.

Bonacchi, M., Harmelin, G., Peris, A., \& Sani, G. (2011). A novel strategy to improve systemic oxygenation in venovenous extracorporeal membrane oxygenation: the " $\chi$-configuration". The Journal of thoracic and cardiovascular surgery, 142(5), 1197-1204. https://doi.org/10.1016/j.jtcvs.2011.01.046.

Bronicki, R. A., Fortenberry, J., Schreiber, M., Checchia, P. A., \& Anas, N. G. (2015). Multicenter randomized controlled trial of inhaled nitric oxide for pediatric acute respiratory distress syndrome. The Journal of pediatrics, 166(2), 365-9.e1. https://doi.org/10.1016/j.jpeds.2014.10.011.

Cabezón et al. (2013). Síndrome de distrés respiratorio agudo: revisióna propósito de la definición de Berlín. Revista Española de Anestesiología y Reanimación, 61(6), 319-327. http://dx.doi.org/10.1016/j.redar.2014.02.007.

Celesia, B. M., Marino, A., Borracino, S., Arcadipane, A. F., Pantò, G., Gussio, M., Coniglio, S., Pennisi, A., Cacopardo, B., \& Panarello, G. (2020). Successful Extracorporeal Membrane Oxygenation Treatment in an Acquired Immune Deficiency Syndrome (AIDS) Patient with Acute Respiratory Distress Syndrome (ARDS) Complicating Pneumocystis jirovecii Pneumonia: A Challenging Case. The American journal of case reports, 21, e919570. https://doi.org/10.12659/AJCR.919570

De Rosa, F. G., Fanelli, V., Corcione, S., Urbino, R., Bonetto, C., Ricci, D., Rinaldi, M., Di Perri, G., Bonora, S., \& Ranieri, M. V. (2014). Extra Corporeal Membrane Oxygenation (ECMO) in three HIV-positive patients with acute respiratory distress syndrome. BMC anesthesiology, $14,37$. https://doi.org/10.1186/1471-2253-14-37

Diehl, A. A. (2004). Pesquisa em ciências sociais aplicadas: métodos e técnicas. São Paulo: Prentice Hall.

Galvão, T. F., \& Pereira, M. G. (2014). Revisões sistemáticas da literatura: passos para sua elaboração. Epidemiologia e Serviços de Saúde, 23(1), 183-184. http://scielo.iec.gov.br/scielo.php?script=sci_arttext\&pid=S1679-49742014000100018\&lng=pt\&tlng=pt.

Grasselli, G., Zanella, A., \& Pesenti, A. (2019). Veno-venous extracorporeal membrane oxygenation in acute respiratory distress syndrome: should the EOLIA Study results change our clinical approach?. Minerva anestesiologica, 85(8), 909-913. https://doi.org/10.23736/S0375-9393.19.13490-6

Hernandez Conte, A. T., Ng, D., Ramzy, D., Dilibero, D., LaBounty, T. M., Gaultier, C., \& Behringer, E. C. (2018). Extracorporeal Membrane Oxygenation in a 29-Year-Old Man with Pneumocystis jirovecii Respiratory Failure and AIDS. Texas Heart Institute journal, 45(4), 254-259. https://doi.org/10.14503/THIJ$16-6186$

Horikita, S., Sanui, M., Fujimoto, Y., \& Lefor, A. K. (2017). Successful repeat ECMO in a patient with AIDS and ARDS. BMJ case reports, 2017, bcr2017219870. https://doi.org/10.1136/bcr-2017-219870

Kim, K. T., Morton, S., Howe, S., Chiew, Y. S., Knopp, J. L., Docherty, P., Pretty, C., Desaive, T., Benyo, B., Szlavecz, A., Moeller, K., Shaw, G. M., \& Chase, J. G. (2020). Model-based PEEP titration versus standard practice in mechanical ventilation: a randomised controlled trial. Trials, 21(1), 130. https://doi.org/10.1186/s13063-019-4035-7

Lee, N., Lawrence, D., Patel, B., \& Ledot, S. (2017). HIV-related Pneumocystis jirovecii pneumonia managed with caspofungin and veno-venous extracorporeal membrane oxygenation rescue therapy. BMJ case reports, 2017, bcr2017221214. https://doi.org/10.1136/bcr-2017-221214

Mauri, T., Grasselli, G., Suriano, G., Eronia, N., Spadaro, S., Turrini, C., Patroniti, N., Bellani, G., \& Pesenti, A. (2016). Control of Respiratory Drive and Effort in Extracorporeal Membrane Oxygenation Patients Recovering from Severe Acute Respiratory Distress Syndrome. Anesthesiology, $125(1)$, $159-167$. https://doi.org/10.1097/ALN.0000000000001103 
Research, Society and Development, v. 10, n. 9, e1210917727, 2021 (CC BY 4.0) | ISSN 2525-3409 | DOI: http://dx.doi.org/10.33448/rsd-v10i9.17727

Nakamura, K., Hikone, M., Shimizu, H., Kuwahara, Y., Tanabe, M., Kobayashi, M., Ishida, T., Sugiyama, K., Washino, T., Sakamoto, N., \& Hamabe, Y. (2020). A sporadic COVID-19 pneumonia treated with extracorporeal membrane oxygenation in Tokyo, Japan: A case report. Journal of infection and chemotherapy: official journal of the Japan Society of Chemotherapy, 26(7), 756-761. https://doi.org/10.1016/j.jiac.2020.03.018

Rieder, M., Schubach, F., Schmoor, C., von Spee-Mayer, C., Wengenmayer, T., Rilinger, J., Staudacher, D., Bode, C., Duerschmied, D., \& Supady, A. (2021). Cytokine adsorption in patients with severe COVID-19 pneumonia requiring extracorporeal membrane oxygenation: protocol for a randomised, controlled, openlabel intervention, multicentre trial. BMJ open, 11(1), e043345. https://doi.org/10.1136/bmjopen-2020-043345

Rilinger, J., Staudacher, D. L., Rieg, S., Duerschmied, D., Bode, C., \& Wengenmayer, T. (2019). Extracorporeal membrane oxygenation in Pneumocystis jirovecii pneumonia: outcome in HIV and non-HIV patients. Critical care (London, England), 23(1), 356. https://doi.org/10.1186/s13054-019-2661-9

Schneider, J. B., Sweberg, T., Asaro, L. A., Kirby, A., Wypij, D., Thiagarajan, R. R., Curley, M., \& Randomized Evaluation of Sedation Titration for Respiratory Failure (RESTORE) Study Investigators (2017). Sedation Management in Children Supported on Extracorporeal Membrane Oxygenation for Acute Respiratory Failure. Critical care medicine, 45(10), e1001-e1010. https://doi.org/10.1097/CCM.0000000000002540

Sousa, M. N. A. (2020). Trilhas acadêmicas: caminhos para a concepção, execução e publicação de artigos científicos. Curitiba: Editora CRV

Stokes, J. W., Gannon, W. D., Sherrill, W. H., Armistead, L. B., Bacchetta, M., Rice, T. W., Semler, M. W., \& Casey, J. D. (2020). Bleeding, Thromboembolism, and Clinical Outcomes in Venovenous Extracorporeal Membrane Oxygenation. Critical care explorations, $2(11)$ e0267. https://doi.org/10.1097/CCE.0000000000000267

Wilcox, M. E., Jaramillo-Rocha, V., Hodgson, C., Taglione, M. S., Ferguson, N. D., \& Fan, E. (2020). Long-Term Quality of Life After Extracorporeal Membrane Oxygenation in ARDS Survivors: Systematic Review and Meta-Analysis. Journal of intensive care medicine, 35(3), 233-243. https://doi.org/10.1177/0885066617737035

Zina, L. G., \& Moimaz, S. A. S. (2016). Odontologia baseada em evidência: etapas e métodos de uma revisão sistemática. Arquivos Em Odontologia, 48(3). https://periodicos.ufmg.br/index.php/arquivosemodontologia/article/view/3610 